\title{
Antenas monopolo de doble banda para captación de energía de radiofrecuencia del medio ambiente
}

\author{
Dual-band monopole antennas for RF energy \\ harvesting from the environment
}

\author{
Edison Zapata, Francisco López, and Germán Goéz
}

\begin{abstract}
This paper presents two low-cost dual-band antennas for collecting ambient RF energy from the GSM-850, GSM-1900, and UMTS-2100 $\mathrm{MHz}$ bands. Both rectennas (Rectifying Antennas) are based on Defected Grounded Structure (DGS) antennas. The first one is designed with a square resonator ring. The bandwidth is between 73.4 and $145 \mathrm{MHz}$, with a gain of 2.29 and $3.53 \mathrm{dBi}$. The other one has a bandwidth of 86 and $124.8 \mathrm{MHz}$ with a gain of 1 and $3.8 \mathrm{dBi}$. In addition, a triple-band rectifier with an HSMS-286C Schottky diode is used to improve the RF to DC power conversion efficiency. Measurement results show that between 150 and $308 \mathrm{mV}$ of $\mathrm{DC}$ is harvested during 8 hours at 50 meters from a telephone base station, in a total of 86 continuous hours of exposure to this station, a DC voltage varying between 4.0 and 4.50 volts of ambient RF energy is obtained.
\end{abstract}

Index Terms - Monopole antenna, dual-band, GMS band, rectifier, energy harvesting.

Resumen - Este artículo presenta dos rectennas (Rectifying Antennas) de bajo costo y de doble banda para recolectar energía de RF de las bandas GSM-850, GSM-1900 y UMTS-2100 MHz. Ambas rectennas se basan en antenas monopolos con plano de tierra de tipo Defected Grounded Structure (DGS). La primera está diseñada con un anillo resonador cuadrado, el ancho de banda se encuentra entre 73.4 y $145 \mathrm{MHz}$, con una ganancia de 2.29 y $3.53 \mathrm{dBi}$. La segunda presenta un ancho de banda de 86 y 124.8 MHz con una ganancia de 1 y $3.8 \mathrm{dBi}$. Además, un rectificador de triple banda con un diodo Schottky HSMS-286C para mejorar la eficiencia de conversión de potencia de RF a CC. Los resultados de la medición muestran que se ha logrado recolectar entre 150 y $308 \mathrm{mV}$ de CC durante 8 horas a 50 metros de una estación base de telefonía, en un total de 86 horas continuas de exposición a esta estación se ha obtenido un voltaje de CC que varía entre 4 V y $4.50 \mathrm{~V}$ de energía de $R F$ ambiental.

Palabras Claves- Antena monopolo, banda GSM, cosecha de energía inalámbrica, doble banda, rectificador.

E. Zapata. Estudiante MSc. Automatización y Control Facultad de Ingenierías, Grupo Automática, Electrónica y Ciencias Computacionales, Instituto Tecnológico Metropolitano, Medellín, Colombia (email: edisonzapata110337@correo.itm.edu.co).

F. López and G. Goéz son docentes de la Facultad de Ingenierías, Grupo Automática, Electrónica y Ciencias Computacionales, Instituto Tecnológico Metropolitano, Medellín, Colombia, (email:franciscolopez@itm.edu.co, germangoez@itm.edu.co).

\section{INTRODUCCIÓN}

$\mathrm{E}$ $\mathrm{N}$ los últimos años, los avances en la tecnología de dispositivos inalámbricos y sus aplicaciones en redes de sensores y sistemas IoT (Internet Of Things) han sido utilizados para medidores de presión, humedad, temperatura y aplicaciones biomédicas [1]. La tecnología de recolección de energía de radio frecuencia (RF) es una buena opción para la carga de energía de dispositivos de bajo consumo de potencia ya que, uno de los principales problemas que estos dispositivos enfrentan es el reemplazo y mantenimiento de baterías.

La energía de RF se encuentra disponible en el medio ambiente debido a múltiples fuentes emisoras como las estaciones de radio y televisión, estaciones satelitales, internet inalámbrico y transmisiones digitales de multimedia. Aunque, la densidad de potencia de la energía RF del medio ambiente es relativamente pequeña [2], se han diseñado sistemas para la recolección de esta energía. Este sistema consiste en una antena y un circuito rectificador con un diodo Schottky HSMS 2862 para funcionar a una frecuencia de $900 \mathrm{MHz}$, los dos elementos son integrados para recibir la potencia de RF y convertirla en corriente continua (CC), con una potencia de entrada de 0 a $9 \mathrm{~mW}$. Esta combinación se conoce como rectenna (Rectifying Antenna) [3], [4].

En la literatura se reporta una rectenna de tipo fractal para la captación de energía RF en las bandas GSM-1800 MHz, esta rectenna es capaz de alimentar un reloj LCD sin batería a una distancia de 10 metros de la torre celular, el rectificador posee un stub para que este tenga una impedancia de $50 \Omega$ y así se mejore la eficiencia de conversión de RF a CC. La máxima tensión de salida es $1.5 \mathrm{~V}$, este diseño de antena presenta un patrón de radiación omnidireccional con un ancho de banda de $750 \mathrm{MHz}$ para una frecuencia de $2.15 \mathrm{GHz}$ y una ganancia de $2.2 \mathrm{~dB}[5]$.

Una rectenna compacta de $18 \mathrm{~mm}$ x $30 \mathrm{~mm}$ con grosor de $16 \mathrm{~mm}$, es diseñada con ranuras rectangulares para mejorar la impedancia de adaptación entre esta y el circuito rectificador, por lo que logra una ganancia de $5.6 \mathrm{~dB}$. Se utiliza el rectificador de Cockroft-Walton de una sola etapa. Esta antena presenta un patrón de radiación tipo dipolo en el plano $\mathrm{E}$ y 
omnidireccional en el plano $\mathrm{H}$ [5].

Otra rectenna que opera en las bandas GSM-900 y $1800 \mathrm{MHz}$, miniaturizada mediante líneas de meandro, y un rectificador a través de un diodo Schottky SMS-7630 para optimizar el nivel de potencia de entrada de $-20 \mathrm{dBm}$, logran una tensión de salida de 183 - $415 \mathrm{mV}$ [6].

Las ondas electromagnéticas del entorno ambiental son de polarización y ángulos de incidencia aleatorios, debido a esto, las antenas recolectoras de energía RF deben tener un patrón de radiación preferiblemente omnidireccional y una amplia banda de frecuencias de operación. Algunas de las antenas que reúnen estas características son las antenas dipolo, dipolo cruzado y antenas en forma de espiral. Para la captura de energía de RF es ideal que una antena posea polarización circular debido a que posee una ventaja en relación a su alta sensibilidad y recibe fácilmente las ondas electromagnéticas del espacio [7].

Por esta razón, en este estudio, se propone una rectenna y un rectificador de doble banda, de bajo costo y de fácil construcción para la recolección de energía de RF ambiental. El prototipo de rectenna propuesto captura la energía electromagnética del medio ambiente de las señales de difusión de UMTS y GSM, las mismas que presentan niveles típicos de potencia y opera en dos bandas de frecuencia.

\section{DisEÑO DE LAS ANTENAS}

\section{A. Antenas mopolo}

Las antenas monopolo tienen la ventaja de ser pequeñas, fáciles de construir, son de bajo costo y son compatibles con los sistemas de RF. Se han realizado varias investigaciones con este tipo de antenas debido a la utilidad que presentan en los sistemas de comunicaciones y recolección de energía inalámbrica como: WLAN (2484 MHz), WiMAX (3400 MHz, $3690 \mathrm{MHz}$ ), (850 MHz, $1900 \mathrm{MHz}$ ) [6], [8], [9]. Para mejorar las características como ancho de banda, ganancia y hasta para miniaturizar los diseños de las antenas de microcinta se utiliza la inserción de ranuras o muescas. Algunos autores utilizan ranuras en forma de $\mathrm{U}$ [10], V [11], ranuras cuadradas [12] y estructuras DGS [13].

El análisis y modelado de las antenas propuestas se realiza mediante el software de simulación CST Microwave Studio, el cual es un software de análisis EM de onda completa, que cumple con la finalidad de analizar y optimizar componentes y sistemas electromagnéticos. La evolución del diseño de las antenas se presenta en la Fig. 1. En la Fig. 1(a) se observa la estructura inicial monopolo rectangular con plano de tierra completo, la Fig. 1(b) muestra un monopolo rectangular con plano de tierra recortado, la Fig. 1(c) presenta una antena monopolo rectangular con inserción de ranura tipo mancuerna en el plano de tierra recortado y la Fig. 1(d) presenta una antena monopolo con anillo resonador cuadrado en la parte superior y plano de tierra recortado con ranura tipo mancuerna.

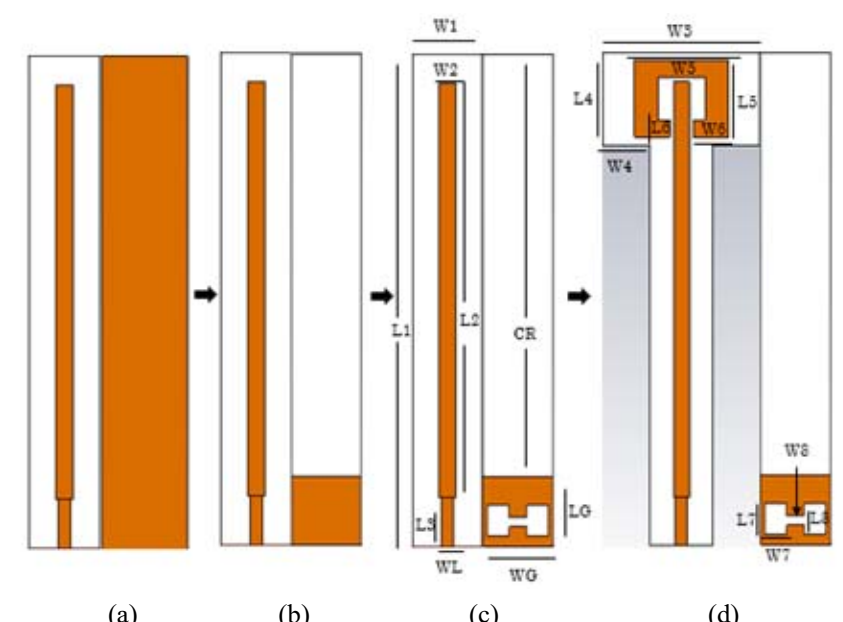

(a)

(b)

(c)

(d)

Fig. 1. Evolución del diseño propuesto para las antenas monopolo. En (a) estructura inicial monopolo rectangular, con plano de tierra completo, (b) monopolo rectangular con plano de tierra recortado, (c) monopolo rectangular con inserción de ranura tipo mancuerna en el plano de tierra recortado y (d) monopolo con anillo resonador cuadrado en la parte superior y plano de tierra recortado con ranura tipo mancuerna

Esta antena es alimentada por una línea de microcinta con impedancia característica igual a $50 \Omega$, el plano de tierra se recorta y se realiza una ranura de tierra defectuosa (DGS) en forma de mancuerna para ajustar la frecuencia deseada y mejorar el patrón de radiación Fig. 1(c) [14]. También, se adiciona un anillo resonador dividido en la parte superior del dipolo Fig. 1(d) para mejorar el ancho de banda y la ganancia de la antena [9].

En el proceso de diseño de antenas de microcinta, investigaciones científicas y experimentales han demostrado que al insertar algún tipo de estructura como anillos resonantes cuadrados o circulares cercanas a la línea de alimentación o al parche radiante de una antena de microcinta, estas generan un cambio en la propagación de las ondas electromagnéticas que viajan por el material de la antena, lo que disminuye las pérdidas de transmisión y aumenta el rango de frecuencias de operación de dicha antena. Por lo que, la abertura del anillo dispone la acumulación de cargas positivas en la mitad superior del anillo, y por defecto, de cargas negativas en la mitad inferior del anillo. De esa manera, los conductores introducen un comportamiento inductivo, que junto con la capacidad eléctrica distribuida entre el anillo produce un comportamiento resonante semejante a un circuito LC [15].

Las características del substrato elegido son: material FR4 constante dieléctrica sugerida 4.3, tangente de perdidas sugerida 0.025 , espesor de dieléctrico igual a $1.6 \mathrm{~mm}$, espesura del cobre igual a $0.035 \mathrm{~mm}$. Mediante el software CST se optimiza el diseño a través de la función de barrido paramétrico para lograr las dimensiones finales. Las dimensiones finales de la antena optimizada propuesta (Fig. 1(d)) se presentan en la Tabla I. Adicionalmente, fotografías de los prototipos de las topologías Fig. 1(c) y Fig. 1(d) construidos, se presentan en la Fig. 2. 
TABLA I

DIMENSIONES DE LAS ANTENAS EN (MM)

\begin{tabular}{cccc}
\hline \hline Parámetro & Dimensión & Parámetro & Dimensión \\
\hline L1 & 116 & L5 & 18 \\
W1 & 16 & W5 & 24 \\
L2 & 97.5 & L6 & 4 \\
W2 & 4 & W6 & 9 \\
L3 & 11.5 & L7 & 5 \\
W3 & 40 & W7 & 5 \\
L4 & 18 & L8 & 1.5 \\
W4 & 12 & W8 & 4 \\
LG & 11 & WG & 16 \\
WL & 3 & CR & 105 \\
\hline \hline
\end{tabular}

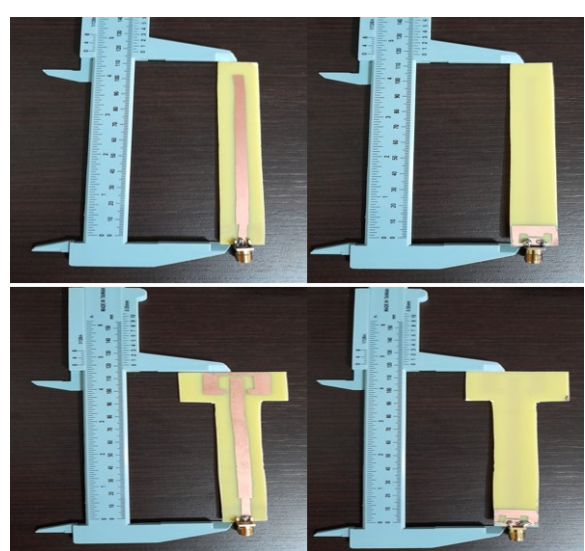

Fig. 2. Fotografías de los prototipos presentados en la Fig.1 (c), parte superior, y Fig. 1(d), parte inferior. Las antenas propuestas se realizaron mediante el método convencional al utilizar papel de impresión e inmersión de la pieza en ácido férrico para retirar el cobre restante de la pieza

Las antenas de microcinta a pesar de tener propiedades de peso ligero, fácil fabricación y ser de gran utilidad en aplicaciones de comunicaciones presentan como limitante un ancho de banda estrecho [16] - [18]. La antena propuesta presenta un ancho de banda entre $5.71 \%$ y $8.18 \%$. Los diagramas de radiación de este tipo de antenas son omnidireccionales o cuasi omnidireccional, pero, generalmente no logran tener ganancias superiores a $3 \mathrm{~dB}$ [19]. La Fig. 3(a) muestra el $\left|S_{11}\right|$ en dB y a partir de esa información se observa que las frecuencias de mejor casamiento son de $890 \mathrm{MHz}$ y $1967 \mathrm{MHz}$. La Fig. 3(b) presenta los patrones de radiación de la antena propuesta en el plano E.

En la Fig. 4(a) se muestra el $\left|S_{11}\right|$ en dB y se observa que las frecuencias de mejor casamiento cuando se agrega el anillo resonador al monopolo son de $870 \mathrm{MHz}$ y $1939 \mathrm{MHz}$. La Fig. 4(b) presenta los patrones de radiación de cada frecuencia de la antena propuesta en el plano E.

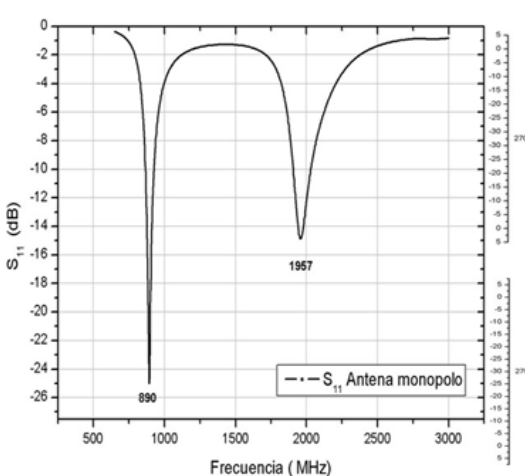

(a)

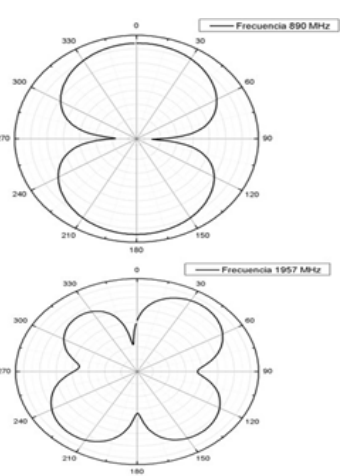

(b)
Fig. 3. (a) Coeficiente de reflexión $\left|S_{11}\right|$ en $\mathrm{dB}$ de la antena monopolo frecuencia 1 en $890 \mathrm{MHz}$ frecuencia 2 en $1957 \mathrm{MHz}$, (b) patrones de radiación en el plano E para cada frecuencia de operación

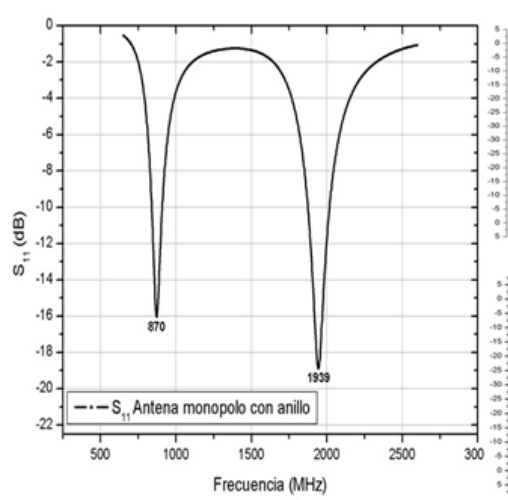

(a)

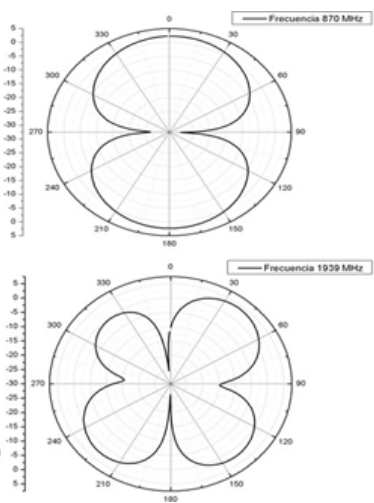

(b)
Fig. 4. (a) Coeficiente de reflexión $\left|S_{11}\right|$ en dB de la antena monopolo con anillo resonante frecuencia lubicada en $870 \mathrm{MHz}$ frecuencia 2 ubicada en $1939 \mathrm{MHz}$, (b) patrones de radiación en el plano E para cada frecuencia de operación

\section{B. Diseño del rectificador}

La topología del rectificador utiliza la tecnología PCB (Printed Circuit Board) debido a que es fácil de integrar con circuitos impresos y con las antenas diseñadas [20]. Otro tipo de rectificadores utilizados para integrar las rectennas son los basados en la tecnología CMOS (Complementary MetalOxide-Semiconductor). Estos rectificadores son compactos y tienen la capacidad de trabajar con bajas potencias de entrada, sin embargo, operan en una sola banda de frecuencia [21]. El rectificador seleccionado (ver Fig. 5) es de fácil de diseño, presenta un $\left|S_{11}\right|$ en dB de doble banda y para su construcción se utiliza el mismo substrato FR4 con el que se diseñan las antenas propuestas.

El rectificador fue diseñado con una red de adaptación de impedancia mediante el uso de una línea de transmisión en forma de $\mathrm{T}$ [22], diodo Schottky HSMS-286C de montaje en superficie para uso de $915 \mathrm{MHz}$ a $5.8 \mathrm{GHz}$. Es ideal para aplicaciones de etiquetas RF/ID y RF, así como para detección de señales grandes, modulación, conversión de RF a CC o duplicación de voltaje. Tiene una sensibilidad de detección de hasta $50 \mathrm{mV} / \mu \mathrm{W}$ a $915 \mathrm{MHz}$, hasta $35 \mathrm{mV} / \mu \mathrm{W}$ a $2.45 \mathrm{GHz}$ y hasta $25 \mathrm{mV} / \mu \mathrm{W}$ a $5.80 \mathrm{GHz}$ [23]. Además, se adicionó un 
filtro pasa bajo compuesto por tres alas triangulares de iguales dimensiones y mismo número de ranuras en forma de cascada con el fin de optimizar el diseño a la frecuencia de operación. La simulación fue realizada con el software CST Studio, donde para las frecuencias de $910 \mathrm{MHz}$ y $2010 \mathrm{MHz}$ se consideró anchos de banda de $85 \mathrm{MHz}$ y $133 \mathrm{MHz}$, respectivamente. Para almacenar la energía rectificada, se utiliza un condensador de 1 faradio tipo $\mathrm{H}$, de doble capa a 5.5V. El diseño del rectificador se muestra en la Fig. 5 mientras que la simulación de los coeficientes de reflexión se presenta en la Fig. 6.
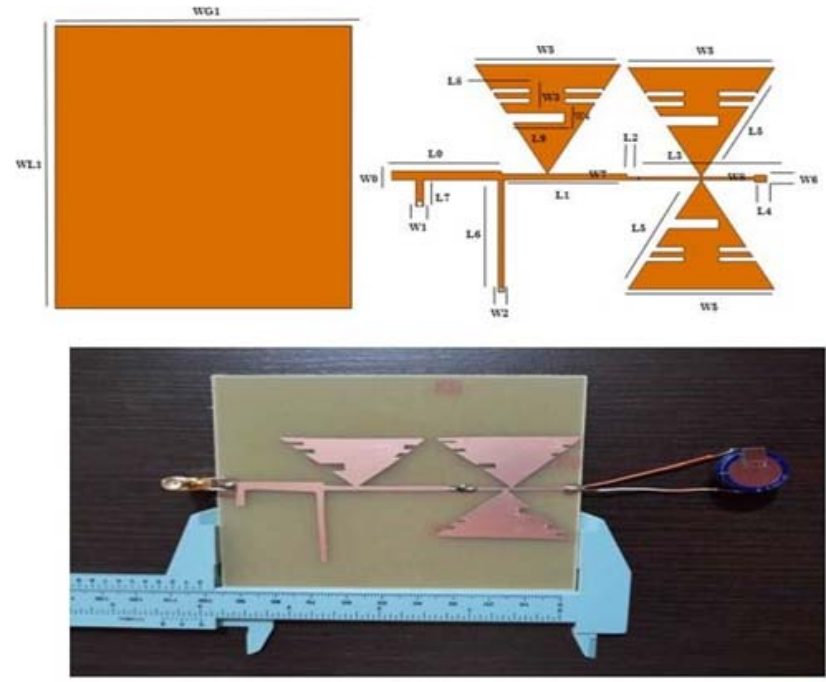

Fig. 5. Diseño y parámetros del rectificador propuesto en $(\mathrm{mm})$ : L0 $=28.7$, $\mathrm{L} 1=35, \mathrm{~L} 2=3.05, \mathrm{~L} 3=30.25, \mathrm{~L} 4=3, \mathrm{~L} 5=37.41, \mathrm{~L} 6=33.80, \mathrm{~L} 7=8$, $\mathrm{L} 8=10, \mathrm{~L} 9=15.55, \mathrm{WL} 1=10, \mathrm{~W} 0=2.64, \mathrm{~W} 1=2, \mathrm{~W} 3=1, \mathrm{~W} 4=2$, $\mathrm{W} 5=38.11, \mathrm{~W} 6=2.20, \mathrm{~W} 7=2.20, \mathrm{~W} 8=1.5, \mathrm{WG} 1=10$

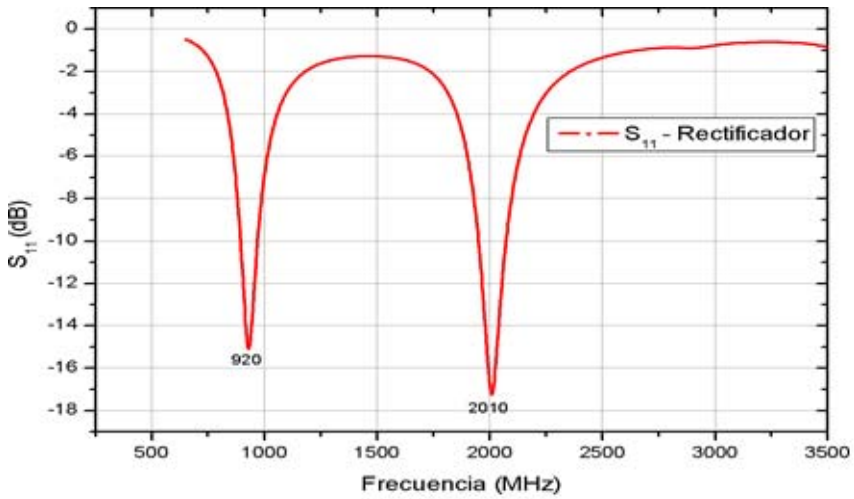

Fig. 6. Simulación del coeficiente de reflexión del rectificador: frecuencia $1=920 \mathrm{MHz}$, con ancho de banda de $85 \mathrm{MHz}$. Frecuencia $2=2010 \mathrm{MHz}$, con ancho de banda de $133 \mathrm{MHz}$

Al realizar ranuras en las alas triangulares del circuito rectificador, la corriente de superficie se desplaza por la estructura lo que cambia la longitud eléctrica del circuito rectificador y, por tanto, incrementa o reduce la frecuencia de trabajo según la necesidad.

\section{Resultados}

Para verificar las bandas de frecuencia y la potencia disipada por la estación base de la cual, se captura la energía de RF, primero se ejecuta un programa para ver el espectro mediante el software GNU Radio [24], una tarjeta USRP 2901 (Universal Software Radio Periphereal - USRP) y una antena comercial de referencia VERT900 (824-960 MHz, 1710-1990 $\mathrm{MHz}$ ) omnidireccional con una ganancia de $3 \mathrm{dBi}$.

El proceso de medida de intensidad de la señal captada se realiza a una distancia de 50 metros de separación entre la estación base y la estación de prueba. Seguidamente, se emplean las antenas propuestas para medir la intensidad de señal recibida. En la Fig. 7, se aprecia el montaje realizado para medir la intensidad de señal. En la Fig. 8(a) y Fig. 8(b) se presentan imágenes del espectro capturado mediante el programa ejecutado con GNU Radio y la USRP.
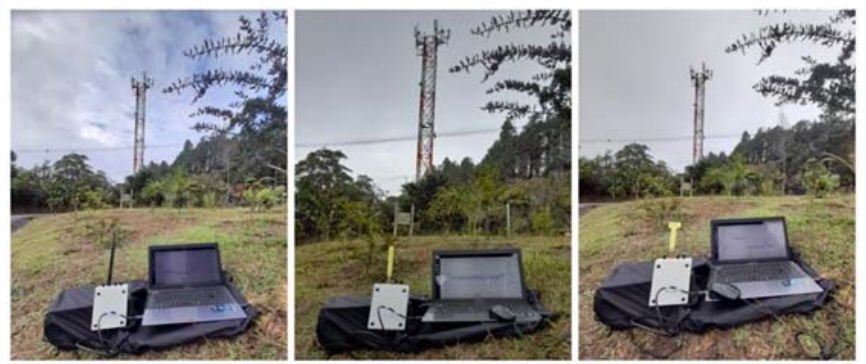

Fig. 7. Medidas de intensidad de la señal izquierda antena VERT900, centro antena monopolo, derecha antena monopolo con anillo dividido

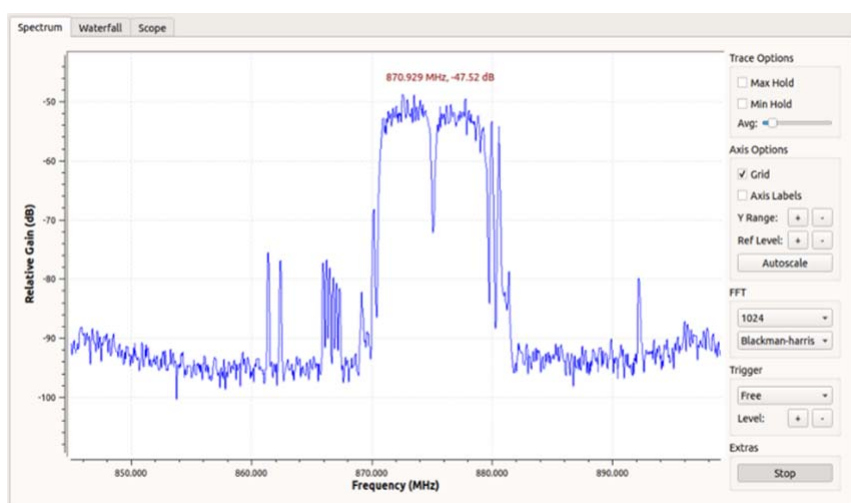

(a)

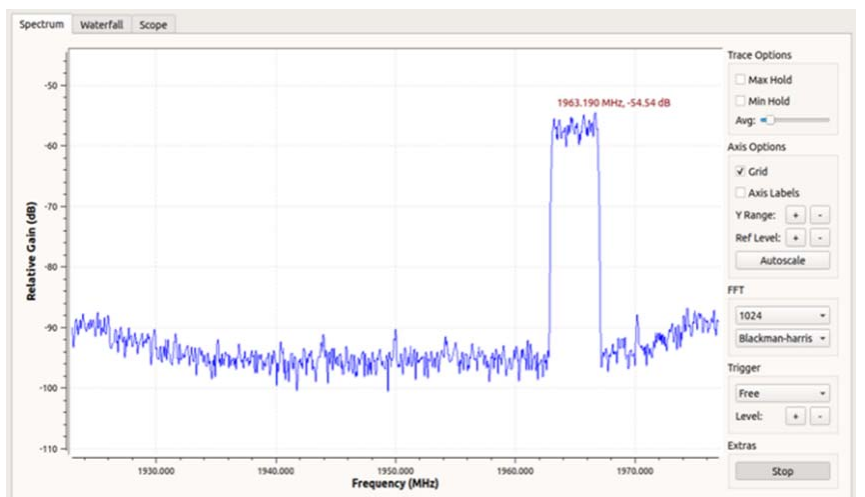

(b)

Fig. 8. Capturas del espectro medido para diferentes frecuencias

En la Tabla II se presenta la comparación de medidas de intensidad de señal recibida por la antena guía y las antenas 
propuestas. En esta medida no se reporta la frecuencia de $2010 \mathrm{MHz}$ debido a que, la antena guía VERT900 solo tiene un rango de $824 \mathrm{MHz}$ hasta $1990 \mathrm{MHz}$.

TABLA II

INTENSIDAD DE LA SEÑAL RECIBIDA EN DBM POR LA ANTENA GUÍA Y LAS ANTENAS PROPUESTAS DESDE LA ESTACIÓN BASE

\begin{tabular}{|c|c|c|}
\hline Antena & Frecuencia & $\begin{array}{c}\text { Intensidad de señal } \\
(\mathrm{dBm})\end{array}$ \\
\hline \multirow{10}{*}{ VERT900 } & $840.2 \mathrm{MHZ}$ & -44.45 \\
\hline & $871.4 \mathrm{MHz}$ & -49.55 \\
\hline & $876.3 \mathrm{MHz}$ & -47.33 \\
\hline & $880.1 \mathrm{MHz}$ & -52.25 \\
\hline & 920.4 MHZ & -70.18 \\
\hline & 950.6 MHZ & -70.90 \\
\hline & $1800 \mathrm{MHZ}$ & -78.48 \\
\hline & $1900 \mathrm{MHZ}$ & -80.82 \\
\hline & $1950 \mathrm{MHZ}$ & -88.76 \\
\hline & $1987 \mathrm{MHZ}$ & -96.55 \\
\hline \multirow{11}{*}{ Monopolo 1} & 871.2 MHZ & -45.53 \\
\hline & 840.3 MHZ & -43.29 \\
\hline & 871.1 MHZ & -46.53 \\
\hline & $876.4 \mathrm{MHz}$ & -46.22 \\
\hline & $880.3 \mathrm{MHz}$ & -51.22 \\
\hline & $1799 \mathrm{MHz}$ & -89.33 \\
\hline & $1900 \mathrm{MHz}$ & -92.23 \\
\hline & $1800 \mathrm{MHZ}$ & -78.48 \\
\hline & $1900 \mathrm{MHZ}$ & -81.67 \\
\hline & $1950 \mathrm{MHZ}$ & -81.59 \\
\hline & $1987 \mathrm{MHZ}$ & -84.63 \\
\hline \multirow{13}{*}{ Monopolo anillo } & $840.4 \mathrm{MHZ}$ & -44.20 \\
\hline & 871.7 MHZ & -47.61 \\
\hline & $872.3 \mathrm{MHz}$ & -39.81 \\
\hline & $880.6 \mathrm{MHz}$ & -53.43 \\
\hline & $1802 \mathrm{MHz}$ & -87.99 \\
\hline & $1883 \mathrm{MHz}$ & -81.99 \\
\hline & $1884 \mathrm{MHz}$ & -88.98 \\
\hline & $1899 \mathrm{MHz}$ & -97.94 \\
\hline & $1900 \mathrm{MHz}$ & -93.44 \\
\hline & $1800 \mathrm{MHZ}$ & -81.26 \\
\hline & $1900 \mathrm{MHZ}$ & -84.13 \\
\hline & $1950 \mathrm{MHZ}$ & -86.25 \\
\hline & $1987 \mathrm{MHZ}$ & -86.76 \\
\hline
\end{tabular}

Las antenas propuestas en conjunto con el rectificador (rectennas) fueron posicionadas a 50 metros de distancia de la estación base ubicada en una zona rural de la cuidad con el fin de restringir a una sola fuente emisora de RF. Aunque, esto es prácticamente improbable ya que el medio ambiente está rodeado de estaciones bases. Adicionalmente, se presentan la Fig. 9(a) y Fig. 9(b), donde se muestran las condiciones ambientales tomadas en un periodo de 8 horas durante la toma de medidas de captación de energía de RF. Los datos de las condiciones ambientales registrados fueron tomados del sistema weather.com para la zona de la ciudad de Medellín-Colombia. En el desarrollo de los ensayos, las condiciones ambientales fueron favorables, no se presentaron tormentas ni precipitaciones que hubieran podido generar algún fallo en la captación de la energía de RF.

Así mismo, durante 8 horas continuas que la rectenna fue expuesta a la radiación de energía $\mathrm{RF}$, emitida por la estación, se logró recolectar $150 \mathrm{mV}$ con la antena monopolo sencilla y $308 \mathrm{mV}$ con la antena monopolo con anillo dividido. Luego de 86 horas continuas de exposición a la radiación de esta estación y, probablemente por otras estaciones circundantes. El prototipo logró una carga de $4.51 \mathrm{~V}$ para la rectenna monopolo y $4.56 \mathrm{~V}$ para la rectenna con anillo recortado, ver Fig. 10 .

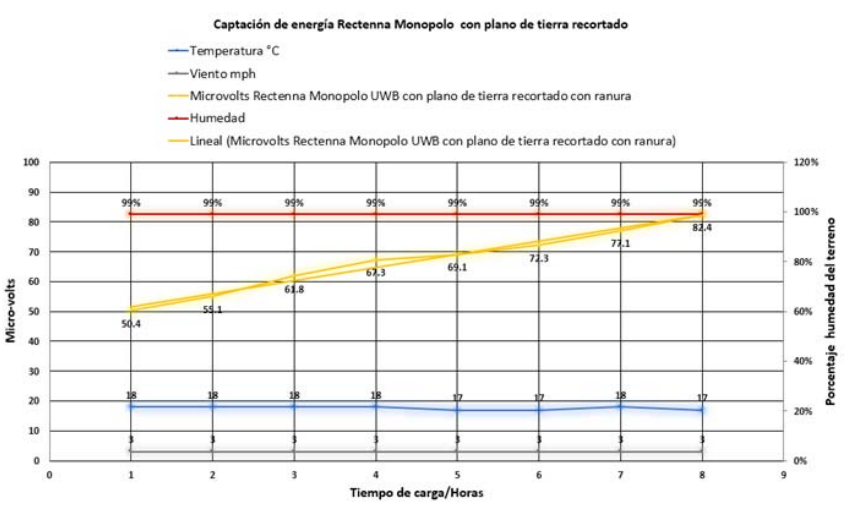

(a)

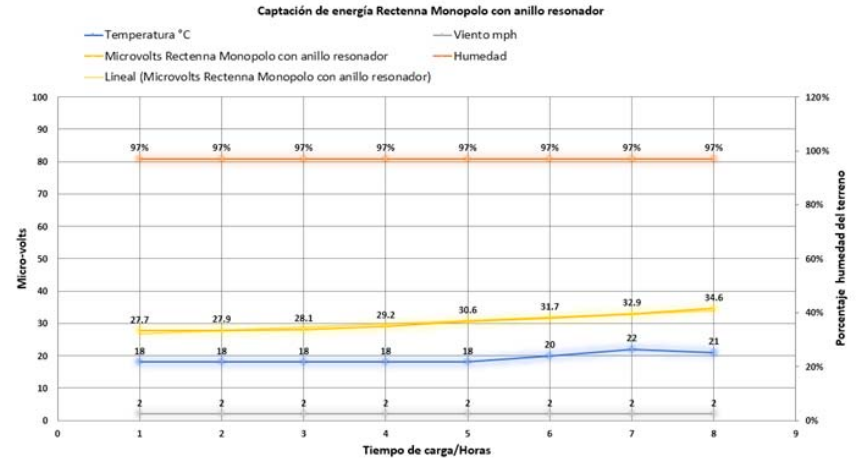

(b)

Fig. 9. Condiciones ambientales durante 8 horas de captación de energía de RF. (a) Rectenna monopolo. (b) Rectenna monopolo con anillo

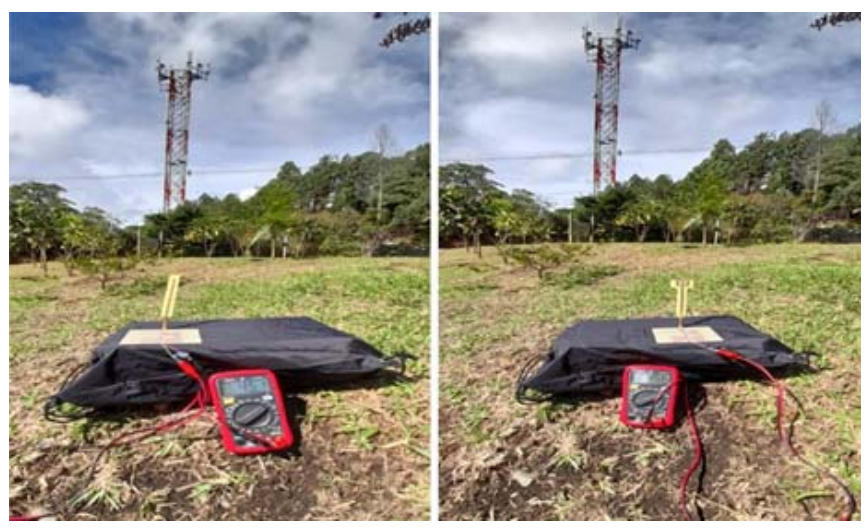

Fig. 10. Captación de energía RF mediante rectennas. Izquierda rectenna monopolo. Derecha antena monopolo con anillo dividido

Ambas rectennas han mostrado buenos resultados para acumular energía de RF, sin embargo, el tiempo de carga es considerablemente extenso para lograr recolectar la cantidad de voltios antes mencionados. Los prototipos de rectennas propuestos podrían ser utilizados en sensores remotos de bajo consumo de potencia. Sin embargo, se debe optimizar el circuito rectificador con objetivo de disminuir sus dimensiones. 


\section{CONCLUSIONES}

Se ha demostrado que se pueden construir antenas multi banda eléctricamente pequeñas para cubrir las bandas GSM y utilizarlas como captadoras de energía. Según los resultados obtenidos las rectennas propuestas podrían suministrar energía para sensores remotos, a largo plazo, pueden suministrar cargas para pequeñas baterías. A una distancia de 50 metros entre la fuente emisora de energía RF y las rectennas, en un tiempo de 86 horas se logró la recolección de $4.51 \mathrm{~V}$ y $4.56 \mathrm{~V}$ que pueden alimentar dispositivos de bajo consumo de potencia como los detectores de humo, medidores, detectores de $\mathrm{CO}$, medidores de gas, Led y otros dispositivos que consuman energía en el orden de los $\mu \mathrm{W}$. Como trabajo futuro se investigarán diseños del rectificador para construirlos más compactos y livianos, mediante la evaluación de substratos de baja pérdida para conducir a un diseño más eficiente.

\section{REFERENCIAS}

[1] K. Shafique et al., "Energy Harvesting Using a Low-Cost Rectenna for Internet of Things (IoT) Applications," IEEE Access, vol. 6, pp. 3093230941, May. 2018.

[2] L.-G. Tran, H.-K. Cha, and W.-T. Park, "RF power harvesting: a review on designing methodologies and applications," Micro Nano Syst. Lett., vol. 5, no. 1, p. 14, Feb. 2017.

[3] K. Kaviarasu and V. Ganesh, "Design and simulation of a $900 \mathrm{MHz}$ rectifier for Rectenna application," in Proc. International Conference on Communications and Signal Processing (ICCSP), Melmaruvathur, India Apr. 2015, pp. 754-756.

[4] A. Okba, S. Charlot, P. F. Calmon, A. Takacs, and H. Aubert, "Multiband rectenna for microwave applications," in Proc. IEEE Wireless Power Transfer Conference (WPTC), Aveiro, Portugal, May. 2016, pp. 1-4.

[5] Q. Awais, Y. Jin, H. T. Chattha, M. Jamil, H. Qiang, and B. A. Khawaja "A compact rectenna system with high conversion efficiency for wireless energy harvesting," IEEE Access, vol. 6, pp. 35857-35866, Jun. 2018.

[6] D. K. Ho, I. Kharrat, V. D. Ngo, T. P. Vuong, Q. C. Nguyen, and M. T. Le, "Dual-band rectenna for ambient RF energy harvesting at GSM 900 $\mathrm{MHz}$ and $1800 \mathrm{MHz}$," in Proc. IEEE International Conference on Sustainable Energy Technologies (ICSET), Hanoi, Vietnam, Nov. 2016 , pp. 306-310.

[7] C. Song et al., "A Novel Six-Band Dual CP Rectenna Using Improved Impedance Matching Technique for Ambient RF Energy Harvesting," IEEE Trans. Antennas Propag., vol. 64, no. 7, pp. 3160-3171, Jul. 2016.

[8] T. A. Elwi and H. S. Ahmed, "A UWB Monopole Antenna Design based RF Energy Harvesting Technology," in Proc. Third Scientific Conference of Electrical Engineering (SCEE), Baghdad, Iraq, Dec. 2018, pp. 111-115.

[9] M. M. Fakharian, "A Wideband Rectenna Using High Gain Fractal Planar Monopole Antenna Array for RF Energy Scavenging," Int. J. Antennas Propag., Jun. 2020.

[10] Y. J. Cho, K. H. Kim, S. H. Hwang, and S. O. Park, "A miniature UWB planar monopole antenna with $5 \mathrm{GHz}$ band-rejection filter," in Proc. The European Conference on Wireless Technology, Parice, France, Oct. 2005, pp. 511-514.

[11] K. Chung, J. Kim, and J. Choi, "Wideband microstrip-fed monopole antenna having frequency band-notch function," IEEE Microw. Wirel. Components Lett., vol. 15, no. 11, pp. 766-768, Nov. 2005.

[12] S. Hu et al., "Backscattering cross section of ultrawideband antennas," IEEE Antennas Wirel. Propag. Lett., vol. 6, pp. 70-73, Mar. 2007.

[13] S. Soltani, M. Azarmanesh, P. Lotfi, and G. Dadashzadeh, "Two novel very small monopole antennas having frequency band notch function using DGS for UWB application," AEU - International Journal of Electronics and Communications, vol. 65, no. 1, pp. 87-94, Jan. 2011.
[14] M. K. Khandelwal, B. K. Kanaujia, and S. Kumar, "Defected ground structure: fundamentals, analysis, and applications in modern wireless trends," Int. J. Antennas Propag., Feb. 2017.

[15] A. E. Hidalgo and F. M. Rizo, "Microstrip antenna with metamaterial hybrid structure for $2.4 \mathrm{GHz}$," Revista de la Facultad de Ingeniería, vol. 27, no. 1, pp. 1-18, Jul. 2021.

[16] M. Karaaslan, M. Bağmanc1, E. Ünal, O. Akgol, and C. Sabah, "Microwave energy harvesting based on metamaterial absorbers with multi-layered square split rings for wireless communications," Opt. Commun., vol. 392, pp. 31-38, Jun. 2017.

[17] A. Estévez Hidalgo, F. Marante Rizo, "Aumento del Ancho de Banda en Antenas de Microcintas a 2,4 GHz con Inserción de Metamateriales," Ingeniería Electrónica, Automática y Comunicaciones, vol. 39, no. 1, pp. 1-15, Mar. 2018.

[18] M. J. Ammann and Z. N. Chen, "Wideband monopole antennas for multi-band wireless systems," IEEE Antennas Propag. Mag., vol. 45, no. 2, pp. 146-150, Apr. 2003.

[19] P. V. Anob, K. P. Ray, and G. Kumar, "Wideband orthogonal square monopole antennas with semi-circular base," in Proc. IEEE Antennas and Propagation Society International Symposium. 2001 Digest. Held in conjunction with: USNC/URSI National Radio Science Meeting (Cat. No.01CH37229), Boston, MA, USA, Jul. 2001, pp. 294-297.

[20] J. J. Lu, X. X. Yang, H. Mei, and C. Tan, "A Four-Band Rectifier with Adaptive Power for Electromagnetic Energy Harvesting," IEEE Microw. Wirel. Components Lett., vol. 26, no. 10, pp. 819-821, Oct. 2016.

[21] C. J. Li and T. C. Lee, "2.4-GHz high-efficiency adaptive power," IEEE Transactions on Very Large Scale Integration (VLSI) Systems, vol. 22, no. 2, pp. 434-438, Feb. 2014.

[22] M. A. Nikravan and Z. Atlasbaf, "T-section dual-band impedance transformer for frequency-dependent complex impedance loads," Electron. Lett., vol. 47, no. 9, pp. 551-553, Apr. 2011.

[23] H. Takhedmit et al., "A 2.45-GHz dual-diode RF-to-dc rectifier for rectenna applications," in Proc. The 40th European Microwave Conference, Paris, France, Sep. 2010, pp. 37-40.

[24] A. F. B. Selva, A. L. G. Reis, K. G. Lenzi, L. G. P. Meloni, and S. E. Barbin, "Introduction to the software-defined radio approach," IEEE Lat. Am. Trans., vol. 10, no. 1, pp. 1156-1161, Jan. 2012. 